\title{
Geriatric neurosurgery: Analysis of a single tertiary centre in Hong Kong
}

\author{
Robert SK Chan*, Calvin HK Mak, TS Tse, FC Cheung and HM Chiu \\ Department of Neurosurgery, Queen Elizabeth Hospital, Hong Kong
}

\begin{abstract}
The elderly aged 80 years and older represent a rapidly growing proportion of Hong Kong's population. It was 3.1\% in 2007 and has increased to $4.9 \%$ in 2017 . It is expected to further raise to $10 \%$ in 2037 .

A retrospective cross-sectional study was conducted for all neurosurgery admission of aged 80 years and above in Queen Elizabeth Hospital from 1 st January 2007 to 31st December 2016. Data was retrieved from Clinical Management System from Hospital Authority. Patient demographics, principal diagnosis, operation performed, perioperative complications, length of stay and mortality were collected and analyzed.

A total of 2611 geriatric admissions were included, which accounted for $11.9 \%$ of all neurosurgery admission in that period. The majority (51\%) of the elderly were admitted for head trauma. There were 397 elderly patients (15.2\%) underwent neurosurgical operations. About 1/8 of elderly patients succumbed during the index hospital stay.

When we dichotomized this 10-year period, our elderly population increased from 3.5\% to 4.5\%, however our geriatric admission percentage doubled from $7.8 \%$ to $15 \%$. There was increased rate of performing neurosurgical operations on the elderly, from $9.3 \%$ rose up to $17.5 \%(\mathrm{p}=0.129)$. The operation mortality rate dropped from $11.8 \%$ to $10.3 \%$ ( $\mathrm{p}=0.157$ ) and their average length of stay reduced from 38.9 days to 25 days ( $\mathrm{p}=0.368)$.

There was increasing demand for elderly neurosurgical care in the setting of growing geriatric population in Hong Kong. They were mostly trauma related. Nearly one-fifth of them required neurosurgical interventions. In recent years, we could achieve lower operation mortality rate and shorter length of stay.
\end{abstract}

\section{Introduction}

As in many big cities in the world, ageing population is a huge social phenomenon facing in Hong Kong. The proportion of elderly population aged greater than 80 rose from $3.3 \%$ in 2007 to $5 \%$ in 2017 [1]. This ageing trend is expected to continue and the pace will become faster in the coming decades. It was projected that the elderly proportion will reach nearly $10 \%$ in 2037 [2] (Figure 1).

In addition, advances in general medical care, diagnostic and surgical techniques were achieved in recent decades. These made the current neurosurgical practice safer and more efficient. The morbidity and mortality to some fragile elderly with multiple medical comorbidities can therefore be reduced. Novel targeted therapies in both primary and metastatic brain tumours also improved the disease control significantly.

Local evidence is needed to evaluate the current burden of this age group in our neurosurgical care as well as whether our interventions improve their survival. The aim of this paper is to retrospectively analyze the diagnosis, procedures and outcomes of these elder neurosurgical patients, as well as any trend variations over time, so that it may help decision making in future clinical resources allocation planning.

\section{Methods}

A retrospective cross-sectional study was conducted for patients aged 80 years and above, who were admitted as inpatients to neurosurgery wards of Queen Elizabeth Hospital from 1st January 2007 to 31st December 2016. Data was retrieved from Clinical Data Analysis and Reporting System in Clinical Management System from Hospital Authority. Details included patient demographics, principal diagnosis, operations performed and length of stay were collected and analyzed. Mortality was defined as death occurred during index hospital stay.

Statistical analysis was performed using SPSS Statistics 24 for Windows. Two-tailed independent t-tests were performed for continuous variables, and Pearson's chi-square tests for categorical variables, with a significance level at $5 \%$.

\section{Results}

During the 10-year period, the total number of patients admitted to neurosurgery wards was 19,309 . Among them, 2,611 (11.9\%) were aged 80 years and over (median age, 84 years). There is slight female predominance in geriatric admission with female-to-male ratio of 1.2:1. Majority of the cases (99.1\%) was from emergency admission.

The most common neurosurgical condition that warranted admission was head trauma, which accounted for $83 \%$, followed by stroke (11\%) and brain tumour (3\%). There were 397 elderly patients (15.2\%) required neurosurgical operations. About $1 / 8$ of elderly patients

${ }^{\star}$ Correspondence to: Robert SK Chan, Department of Neurosurgery, Queen Elizabeth Hospital, Hong Kong, E-mail: lwrobert@hotmail.com

Key words: geriatrics, elderly, ageing, octogenarians, neurosurgery, admission, surgical outcome

Received: April 27, 2019; Accepted: June 10, 2019; Published: June 13, 2019 
died from primary neurosurgical disease or various medical problems during the index hospital stay, whereas the general mortality rate was 4.6\%. The most frequent perioperative complication was pneumonia (37.2\%), followed by urinary tract infection (10.7\%).

In order to study any trend variations over time, the entire 10year study period was dichotomized into two periods: 2007 - 2011 and 2012 - 2016: According to the data from the Census and Statistics Department of Hong Kong, the average baseline geriatric population increased from $3.6 \%$ to $4.5 \%$ across the two periods [1]. However, the proportion of elderly admission doubled from $8.4 \%$ to $17.7 \%(\mathrm{p}=0.157)$ (Figure 2). The overall mortality rate in the elderly group dropped slightly from $13.7 \%$ to $11.8 \%$ ( $\mathrm{p}=0.526)$. The rate of the elderly admitted to neurosurgery ward receiving neurosurgical operations nearly doubled, from $9.3 \%$ rose up to $17.5 \%(\mathrm{p}=0.129)$.

Among the operated cases, the majority (86.7\%) was performed as emergency operations. The main neurosurgical operations performed were burr hole drainage, craniotomy or craniectomy and external ventricular drainage, which composed of nearly $80 \%$ of all operations in this age group (Figure 3 ). The 30-day post operation mortality rate dropped from $11.8 \%$ to $10.3 \%(\mathrm{p}=0.157)$ across the two periods. Their average length of stay also reduced from 38.9 days to 25 days $(\mathrm{p}=0.368)$ (Table 1$)$.

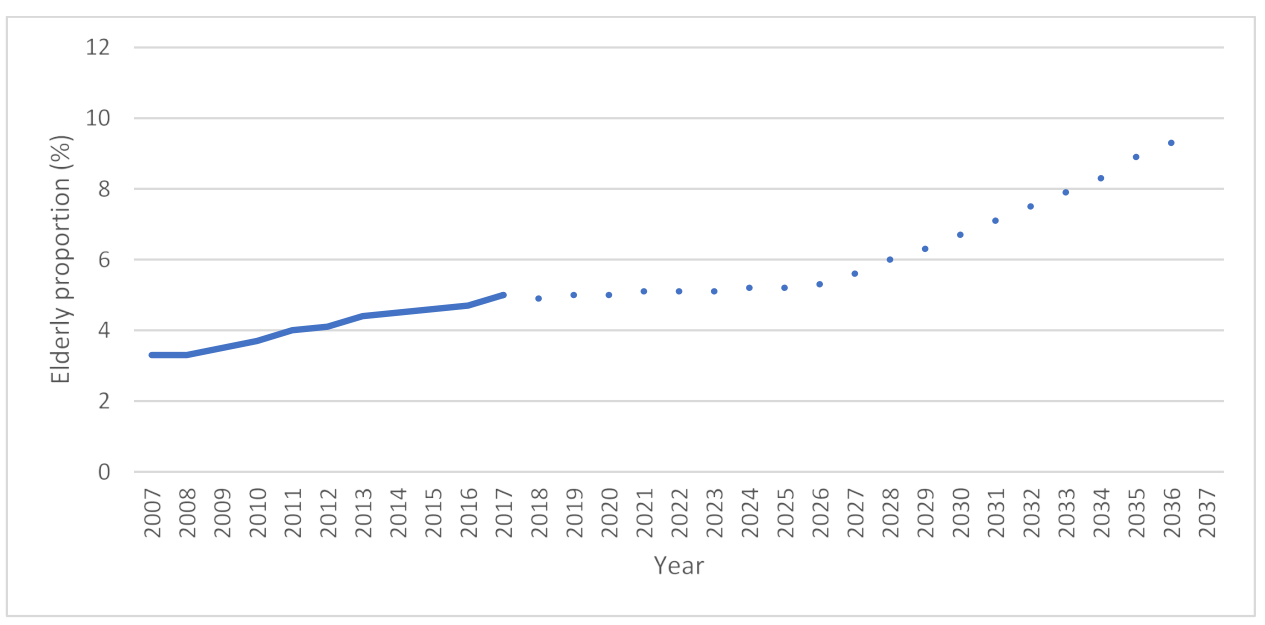

Figure 1. Proportion of elderly in Hong Kong from 2007 to 2037 (projected)

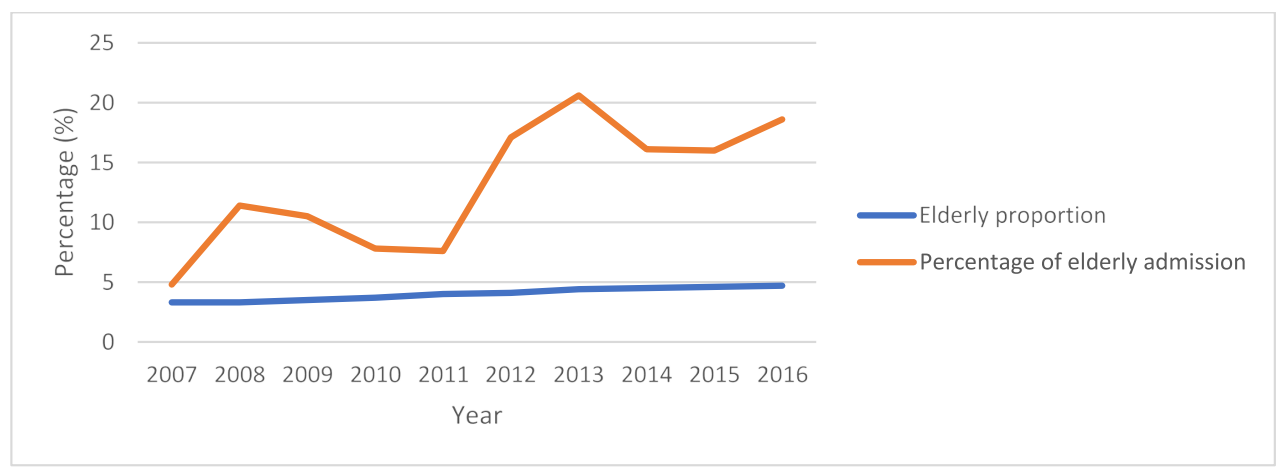

Figure 2. Percentage of elderly admission and Hong Kong elderly proportion during 2007-2016

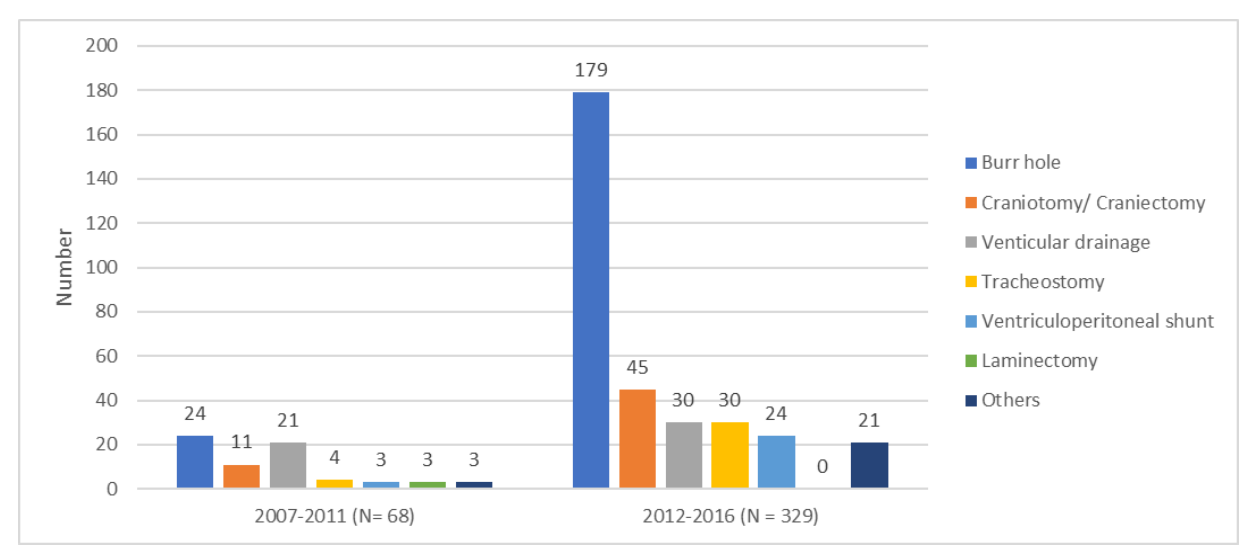

Figure 3. Types of neurosurgical operations performed 
Table 1. Operation statistics from 2007-2016

\begin{tabular}{|c|c|c|c|}
\hline & $\mathbf{2 0 0 7 - 2 0 1 1}$ & $\mathbf{2 0 1 2 - 2 0 1 6}$ & p-value \\
\hline Number & 68 & 329 & 0.267 \\
\hline Emergency operation & $91 \%$ & $83.60 \%$ & 0.317 \\
\hline Operation mortality & $11.80 \%$ & $10.30 \%$ & 0.157 \\
\hline Average length of stay (days) & 38.9 & 25 & 0.368 \\
\hline
\end{tabular}

\section{Discussion}

In parallel with the ageing of the population worldwide [3], the incidence of many neurosurgical diseases such as chronic subdural haematoma increases in elderly patients [4]. These result in an increase in neurosurgical demand within the elderly.

In addition, there are advances in general medical care, such as geriatric medicine and anaesthetic techniques [5,6]. Technology advancement in various domains, such as the use of intraoperative neurophysiological monitoring, stereotactic radiosurgery and development of various endovascular adjuncts, has made neurosurgical practice safer and more efficient [7-11]. Pharmaceutical breakthroughs also brought promising disease control and survival outcome in brain tumour patients [12]. These progresses changed the age limits for neurosurgical interventions and contributed to the increasing likelihood of the elderly patients receiving neurosurgical operations.

The growing demand of neurosurgery care in geriatric population was well observed in other parts of the world. A recent study from González-Bonet et al. found a considerable increase of $77.5 \%$ in geriatric neurosurgery admission in Spain [13].

Comparing our local data with similar international studies concerning neurosurgical care in the elderly, our centre was a high volume one receiving an average of 261 elderly neurosurgery admission each year. There was about one octogenarian in every eight neurosurgery patients. This proportion was similar to $14 \%$ from United Kingdom dataset [14].

In fact, the actual situation may be underestimated because some of the elderly with poor performance status or multiple medical comorbidities were often screened out in Accident and Emergency Department or other clinical departments if limited benefits from neurosurgical interventions were expected. It implies that the genuine demand of neurosurgery care in the elderly was far beyond our results shown here.

The elderly mortality rate of $12.5 \%$ in our series was higher than $4.1 \%$ in Switzerland group [15] and 5.4\% in United Kingdom group [14]. This may be due to the differences in selection bias guided by different cultural background, clinical judgement or logistic reasons. Besides, many elderly deconditioned due to the primary neurological insults or complications developed during the hospital stay [15]. However there is a lack of palliative or infirmary services in Hong Kong to take care of those severely disabled elderly whose family could not afford private nursing homes [16]. It is not a rare situation that some of them needed to spend their end of life in an acute neurosurgical centre [17].

Surgical decision making is a multifactorial process in which age should be considered with other parameters such as Karnofsky Performance Status (KPS) or modified Rankin Scale (mRS) score. Calendar age should be differentiated from biological age. Age alone should not serve as a contraindication for neurosurgery and a selected group of elderly patients can undergo neurosurgical interventions safely [15]. The majority of our neurosurgical operations were for traumatic brain injury (74.5\%). For instance, elderly with chronic subdural haematoma and midline shift was usually managed with burr hole drainage unless medically unfit. Those with good premorbid medical and functional status admitted for acute subdural haematoma or contusion with low Glasgow Coma Scale or midline shift would be offered craniotomy if family was keen for surgical management after acknowledging the likelihood of vegetative or severely disabled status.

The operative rate in the elderly aged 75 years and older from Whitehouse et al. was $88 \%$ from their 4-year retrospective study [14], which was substantially higher than our figure of $17.5 \%$. This may be explained by the broader cut-off age of their geriatric subjects. Besides, different disease composition was observed. Majority of our neurosurgical operations were for traumatic brain injury, whereas the top diagnosis in Whitehouse et al. was degenerative spine diseases (41.4\%) [14].

Another main discrepancy was the proportion of emergency admission. Nearly $99 \%$ of our elderly neurosurgery admission was emergency one, whereas it only accounted for $49.6 \%$ in Whitehouse et al. [14]. The presenting pathology, the associated pathophysiologic stresses, and the complications associated with inpatient hospital care all contribute to the high mortality rate for elderly patients admitted as neurosurgical emergencies.

Future projections of local care provision for elderly neurosurgical patients can be estimated based on our study. In average, the geriatric bed days in our neurosurgical wards increased $21 \%$ annually based on our 10 year results. Based on this model, it is expected that $60 \%$ of our unit's neurosurgical beds will be occupied by elderly patients in 10 years. Therefore, prompt attention is required to improve our inpatient and community postoperative rehabilitation programmes. In addition, more resources should be allocated by the government to help solve the long term caring and placement problems in the elderly population.

Multidisciplinary approach should be considered as the further direction to cope with the increasing demand in neurosurgical care towards the elderly. For instance, shared care between neurosurgeons and geriatricians may be adopted [18]. Joint effort from various allied health professionals is also crucial for restoring the functions in elderly patients.

There were several limitations of this study. It was a retrospective cross-sectional study over a ten-year period. Therefore a number of confounding factors exist, such as availability of diagnostic imaging facilities, advancement in modern medical technology and improvement in intensive care. Besides, reliable data on clinical outcome after discharge could not be obtained for every patient since some of them were transferred to other clinical departments or infirmary care units and some were lost to follow up.

\section{Conclusion}

This study outlines the demographics and outcomes of the elderly admitted to the neurosurgery department of Queen Elizabeth Hospital in Hong Kong. The pattern variation across the 10 years showed that there was an increasing demand for geriatric neurosurgical care. Nowadays, there was about one octogenarian in every eight neurosurgery patients. Their causes of admission were mostly trauma related and nearly one-fifth of them required neurosurgical interventions. However, functional and performance status should be considered together with calendar age in surgical decision making and patient counselling. 


\section{References}

1. Census and statistics department Hong Kong special administrative region, Hong Kong 2016 Population By-census - Thematic report: Older Persons, 2018.

2. Census and statistics department Hong Kong special administrative region, Hong Kong population projections 2017-2066. 2017.

3. United Nations, World population prospects. Anal Rep III.

4. Chibbaro S, Di Rocco F, Makiese O, Mirone G, Marsella M, et al. (2011) Neurosurgery and elderly: Analysis through the years. Neurosurg Rev 34: 229-233. [Crossref]

5. Treschan TA, Schaefer MS, Subasi L, Kaisers W, Schultz MJ, et al. (2015) Evolution of ventilator settings during general anaesthesia for neurosurgery. Eur J Anaesthesiol 32: 894-896. [Crossref]

6. Pasternak JJ, Lanier WL (2018) Neuroanesthesiology update. J Neurosurg Anesthesiol 30: 106-145. [Crossref]

7. Rothwell PM (2018) Clinical innovation in stroke: getting the simple things right. Lancet Neurol 17: 491-493.

8. Smith W, Furlan A (2016) Brief history of endovascular acute ischemic stroke treatment. Stroke 47: e23-e26.

9. Buttrick SS, Shah AH, Basil GW, Komotar RJ (2017) The future of cranial neurosurgery-Adapting new approaches. Neurosurgery 64: 144-150.

10. Chahidi A, Benali SA (2018) Intraoperative electrophysiological neuro-monitoring in neurosurgery: Preliminary experience, pitfalls, theoretical and practical limitations. Clin Neurophysiol 129: e57.
11. Kim SK, Kim K, Cho C, Bang MS, Shin HI, Phi JH (2018) Intraoperative neurophysiological monitoring: A review of techniques used for brain tumor surgery in children. J Korean Neurosurg Soc 61: 363-375.

12. Brastianos PK, Ippen FM, Hafeez U, Gan HK (2018) Emerging gene fusion drivers in primary and metastatic central nervous system malignancies: A review of available evidence for systemic targeted therapies. Oncologist.

13. González-Bonet LG, Tarazona-Santabalbina FJ, Lizán Tudela L (2016) Neurosurgery in the elderly patient: Geriatric neurosurgery. Neurocirugia 27: 155-166.

14. Whitehouse KJ, Jeyaretna DS, Wright A, Whitfield PC (2016) Neurosurgical care in the elderly: Increasing demands necessitate future healthcare planning. World Neurosurg 87: 446-454.

15. Maldaner N, Sarnthein J, Bozinov O, Regli L, Neidert MC (2018) Neurosurgery in octogenarians: A prospective study of perioperative morbidity, mortality, and complications in elderly patients. World Neurosurg 110: e287-e295.

16. Chung RY, Wong EL, Kiang N, Chau PY, Lau JYC, et al. (2017) Knowledge, attitudes, and preferences of advance decisions, end-of-life care, and place of care and death in Hong Kong. J Am Med Dir Assoc 18: 367. [Crossref]

17. Chan CW, Chui YY, Chair SY, Sham MM, Lo RS, et al. (2014) The evaluation of a palliative care programme for people suffering from life-limiting diseases. $J$ Clin Nurs 23: 113-123. [Crossref]

18. Auerbach D, Wachter RM, Cheng HQ, Maselli J, McDermott M, et al. (2010) Comanagement of surgical patients between neurosurgeons and hospitalists. Arch Intern Med 170: 2004-2010. [Crossref]

Copyright: $(02019$ Chan RSK. This is an open-access article distributed under the terms of the Creative Commons Attribution License, which permits unrestricted use, distribution, and reproduction in any medium, provided the original author and source are credited. 